Submitted to: 8th Int'1 Symposium on Electron Beam Ion Sources

and Traps and their Applications

BNL, Upton, NY; Nov 6-8,2000

\title{
RHIC Status and Requirements ${ }^{*}$
}

\author{
Thomas Roser \\ for the RHIC commissioning team \\ Brookhaven National Laboratory \\ Upton, N.Y. 11973-5000, USA
}

\begin{abstract}
Construction of the Brookhaven Relativistic Heavy Ion Collider (RHIC) was officially completed last year and commissioning has begun. RHIC is the first hadron accelerator and collider consisting of two independent rings. It is designed to operate over a wide range of beam energies and with particle species ranging from polarized protons to heavy ions. An overview of the status of commissioning and operation with gold is given and requirements for possible new ion sources are discussed.
\end{abstract}

\section{SUMMARY OF RHIC HEAVY ION COMMISSIONING AND OPERATION}

After a 9 year construction period the Brookhaven Relativistic Heavy Ion Collider (RHIC) has started its commissioning period. After an initial engineering run last year cool-down of the two $3.8 \mathrm{~km}$ long cryostats containing about 1000 super-conducting magnets started in February 2000 and operation with gold beam, delivered by the injector chain consisting of the Tandem, Booster, and AGS, started in April.

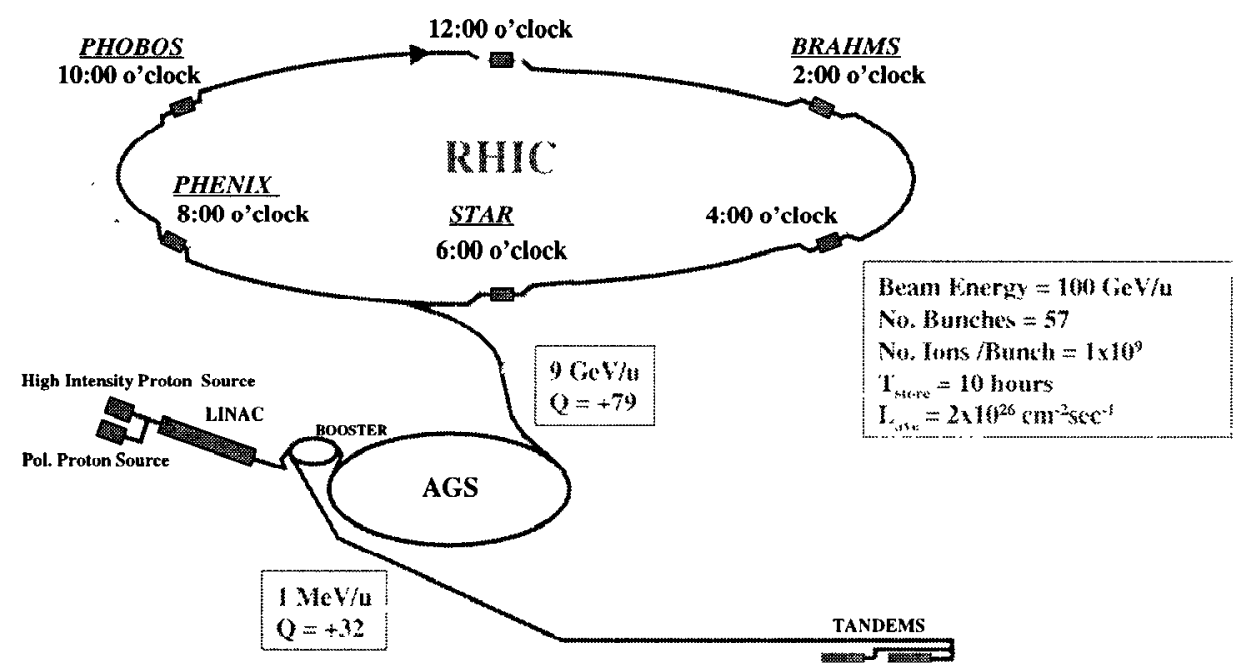

FIGURE 1. Layout of RHIC and the injector accelerators. The gold ions are stepwise ionized as they are accelerated to RHIC injection energy.

\footnotetext{
${ }^{*}$ Work performed under the auspices of the U.S. Department of Energy
} 
Fig. 1 shows the layout of RHIC and the three injector accelerators Tandem, AGS Booster and AGS. The gold ions are stepwise ionized as they are accelerated to RHIC injection energy, at which point they are fully ionized. The performance of the injector is summarized in Table 1. The Tandem Van de Graaff accelerates $\mathrm{Au}^{-1}$ from a sputter source to about $1 \mathrm{MeV} /$ nucleon. The $530 \mu$ s long beam pulse is stripped to $\mathrm{Au}^{+32}$ and injected into the Booster using horizontal and vertical phase space painting. After acceleration to about $100 \mathrm{MeV} /$ nucleon the beam is stripped to $\mathrm{Au}^{+77}$ and transferred to the AGS where it is accelerated to the RHIC injection kinetic energy of $8.6 \mathrm{GeV} /$ nucleon. During acceleration in the AGS the beam bunches from the Booster are merged to reach the required intensity of about $1 \times 10^{9} \mathrm{Au}$ ion per bunch at a longitudinal emittance of $0.3 \mathrm{eVs} /$ nucleon. The final stripping to bare $\mathrm{Au}^{+79}$ occurs on the way to RHIC.

TABLE 1. RHIC injector performance

\begin{tabular}{lcc}
\hline Location & Intensity normalized to a single RHIC bunch & Efficiency \\
\hline Tandem & $3.8 \times 10^{9}$ & \\
Booster Injection & $2.2 \times 10^{9}$ & $58 \%$ \\
Booster Extraction & $1.8 \times 10^{9}$ & $81 \%$ \\
AGS Injection & $0.9 \times 10^{9}$ & $50 \%$ \\
AGS Extraction & $0.9 \times 10^{9}$ & $95 \%$ \\
\hline Total & & $23 \%$ \\
\hline
\end{tabular}

Initial commissioning of RHIC focussed on the magnet power supply control during injection and acceleration. The two RHIC rings, labeled blue and yellow, are intersecting at six interaction regions (IR), four of which are occupied by the collider experiments BRAHMS, STAR, PHENIX and PHOBOS, respectively. To achieve the necessary flexibility to adjust and optimize the collision rate at each interaction region of the two RHIC rings a very large number of independently controllable power supplies were installed and needed to be commissioned. Very precise and reliable beam position monitors were available early on in the commissioning effort and allowed beam-based diagnostics to detect hardware problems. They also allowed us to demonstrate the excellent agreement of the RHIC machine lattice functions with the design.

Table 2 gives the goals for the main machine parameters for the commissioning and first operations period, all of which were either reached or exceeded during the run. The energy at storage of $66 \mathrm{GeV} /$ nucleon is less than the design energy of 100 $\mathrm{GeV} /$ nucleon because the large-bore superconducting dipole magnets used to merge and separate the two beams on either side of the interaction regions require in-situ training to reach their design strength. For this first run this was postponed until the end of the run at which time design performance was achieved successfully.

RHIC is also the first superconducting, slow ramping accelerator that crosses transition energy during acceleration. At transition energy the spreads of the particle revolution frequency stemming from the spread in velocity and spread in path length 
cancel exactly and all particles maintain their relative position for a long time. Interaction between particles can then cause instabilities. Although some beam loss and longitudinal emittance growth was observed in RHIC from crossing transition energy the effect was not excessive due to the still lower beam intensity and the special machine lattice used during this first run that minimized momentum dependent (chromatic) effects at transition. To reach full design intensity of $1 \times 10^{9} \mathrm{Au}$ ions / bunch pulsed quadrupole power supplies will be installed to change transition energy quickly during acceleration to effectively jump across it.

\section{TABLE 2. Parameter goals for RHIC RUN2000}

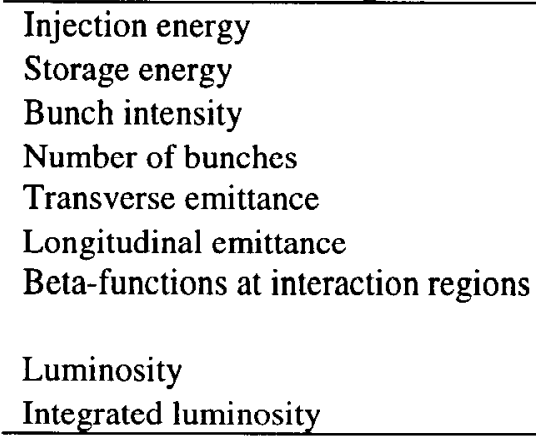

$$
\begin{gathered}
\gamma=10.25(\mathrm{P}=9.5 \mathrm{GeV} / \text { c/nucleon }) \\
\gamma=70.0(\mathrm{P}=65.1 \mathrm{GeV} / \text { /nucleon }) \\
0.5 \times 10^{9} \mathrm{Au} \text { ions } / \text { bunch }
\end{gathered}
$$

56 filled bunches plus 4 empty bunches for abort gap $15 \pi \mu \mathrm{m}$ (normalized, $95 \%$ ) $0.3 \mathrm{eVs} /$ nucleon / bunch $\beta^{*}=3 \mathrm{~m} @ 2,4,8$, and 12 o'clock

$\beta^{*}=8 \mathrm{~m} @ 6$, and 10 o'clock $2 \times 10^{25} \mathrm{~cm}^{-2} \mathrm{~s}^{-1}$ (10\% of design luminosity) a few inverse micro-barns

Fig. 2 shows a typical acceleration cycle which includes filling the blue ring with 56 bunches in groups of 4 bunches, filling the yellow ring in the same way and then simultaneous acceleration of both beams to storage energy. Residual loss of beam that was not captured by the rf system occurs at the beginning of acceleration and from transition crossing about one minute later. Beam losses due to the two beams interacting is also noticeable at injection energy.

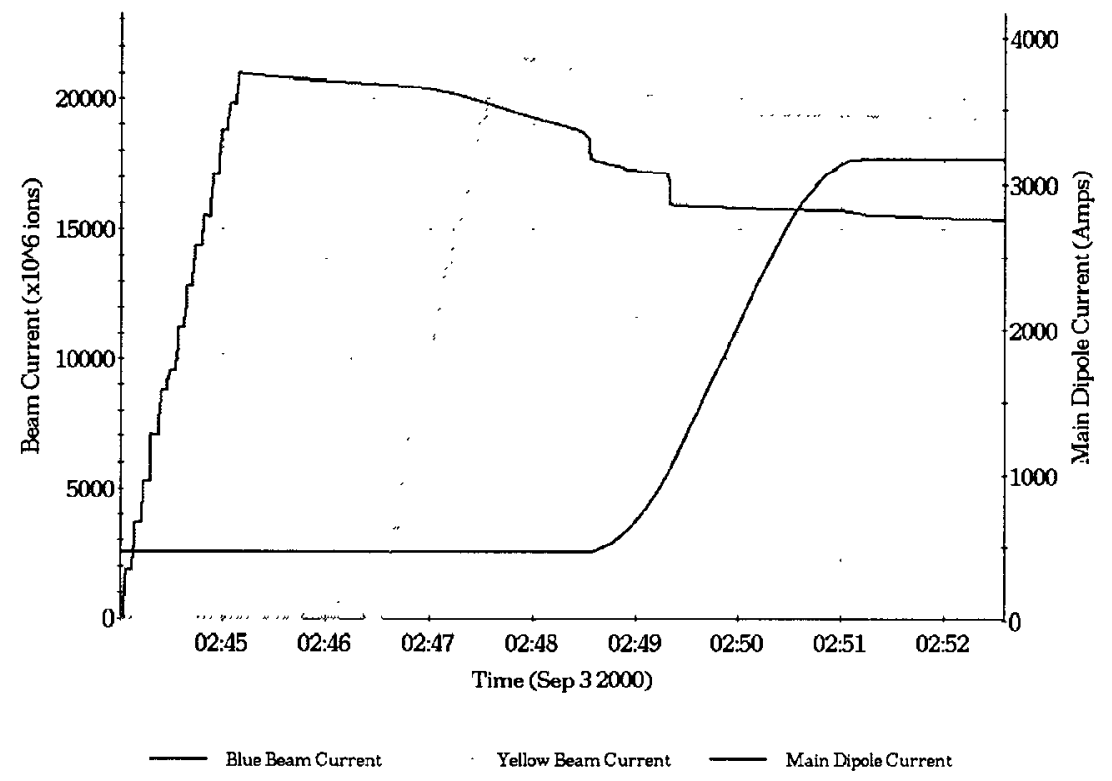

FIGURE 2. Beam intensity evolution during filling and acceleration in RHIC. The dark and light "staircase" lines are the beam intensity in the blue and yellow ring, respectively. The third line shows the main dipole current and is proportional to the beam momentum. 
During the last month all four experiments were in data taking mode and running efficiency at times approached $50 \%$. Typical stores lasted about 5 hours. After longitudinal and transverse steering to optimize collision rate the initial luminosity agreed well with the expected luminosity as calculated from the beam parameters listed in Table 2. Fig. 3 shows the evolution of the collision rate in the four experiments during a typical store. Note that since STAR and PHOBOS are located at the interaction regions with $\beta^{*}=8 \mathrm{~m}$ their collision rates are reduced by a factor of $8 / 3$ from the rates of the other two detectors. The drop of luminosity during the store is due to both beam loss and transverse emittance growth most likely caused by the nonlinearities of the beam-beam interaction and Coulomb (intra-beam) scattering within a bunch. The latter is particularly important for these fully stripped, highly charged gold beams. The integrated luminosity accumulated during the last month exceeded $6(\mu \mathrm{b})^{-1}$ for BRAHMS and PHENIX.

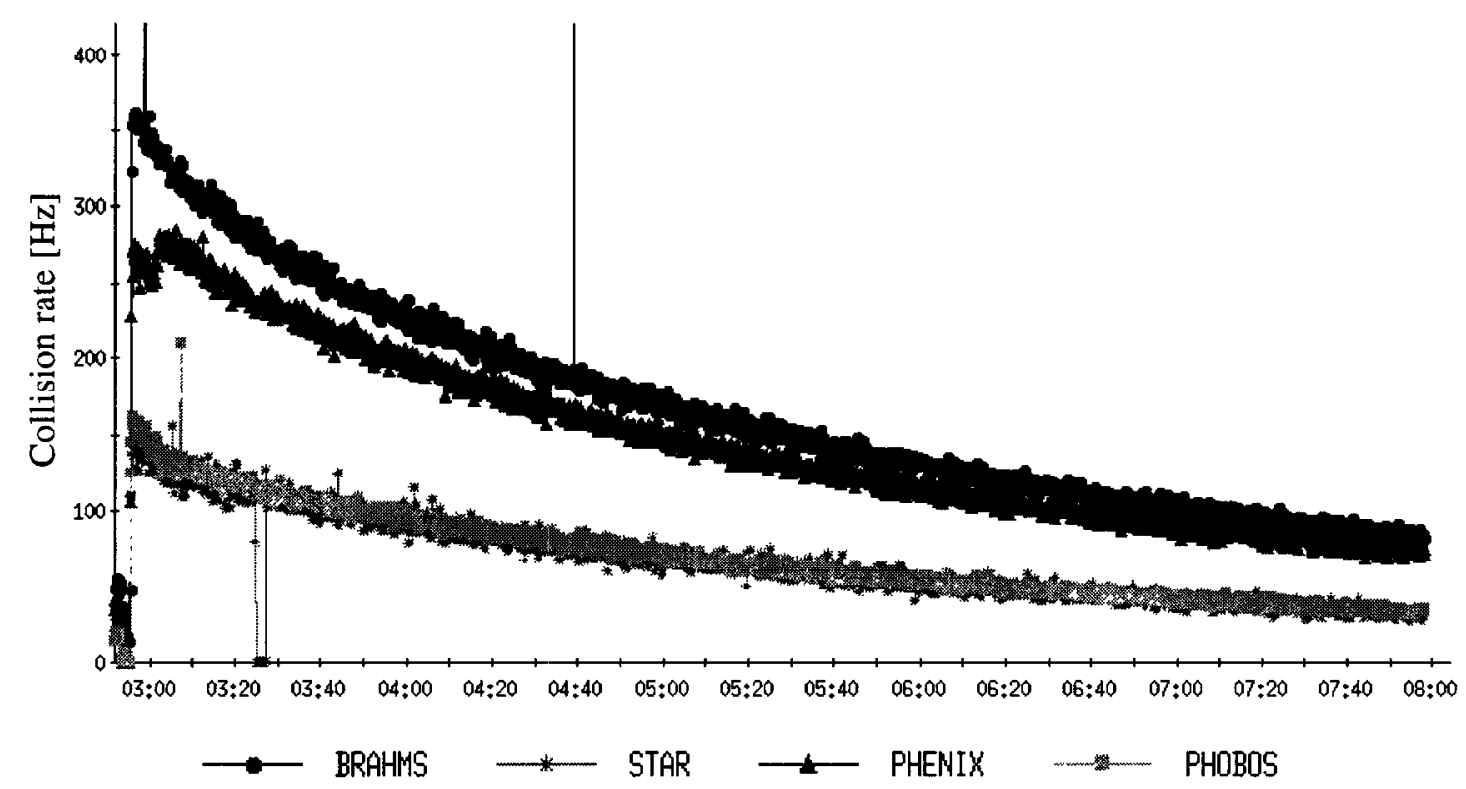

FIGURE 3. Evolution of the collision rate at the four RHIC detectors during a typical store.

\section{PLANS FOR RUN2001}

The performance goal for the upcoming run in 2001 is to reach as much as possible design parameters as summarized in Table 3 . The main new hardware systems consist of power supplies for the transition energy jump, as mentioned above, and a high gradient, high frequency rf system to avoid beam loss from intra-beam scattering during store. 
TABLE 3. Parameter goals for RHIC RUN2001

\begin{tabular}{lc}
\hline Injection energy & $\gamma=10.25(\mathrm{P}=9.5 \mathrm{GeV} / \mathrm{c} /$ nucleon) \\
Storage energy & $\gamma=107.4(\mathrm{P}=100.0 \mathrm{GeV} / \mathrm{c} /$ nucleon $)$ \\
Bunch intensity & $\mathrm{N}=1.0 \times 10^{9} \mathrm{Au}$ ions $/$ bunch \\
Number of bunches & $\mathrm{N}_{\mathrm{b}}=56$ filled bunches plus 4 empty bunches for abort gap \\
Transverse emittance & $\varepsilon=15 \pi \mu \mathrm{m}$ (normalized, $95 \%)$ \\
Longitudinal emittance & $0.3 \mathrm{eVs} /$ nucleon $/$ bunch \\
Beta-functions at injection & $\beta^{*}=10 \mathrm{~m} \mathrm{@} \mathrm{all} \mathrm{interaction} \mathrm{regions}$ \\
Beta-functions at store & $\beta^{*}=2-10 \mathrm{~m} \mathrm{@} \mathrm{all} \mathrm{interaction} \mathrm{regions}$ \\
Luminosity & $2 \times 10^{26} \mathrm{~cm}^{-2} \mathrm{~s}^{-1}$ \\
\hline
\end{tabular}

\section{RHIC UPGRADE PLANS}

The design luminosity in RHIC can be calculated from the parameters given in Table 3 according to the following equation:

$$
L=\frac{3 f_{\text {rev }} \gamma}{2} \frac{N_{b} N^{2}}{\varepsilon \beta^{*}}=9 \ldots 1 \times 10^{26} \mathrm{~cm}^{-2} \mathrm{~s}^{-1} \text { over } 10 \text { hours }
$$

where $f_{\text {rev }}$ is the RHIC revolution frequency of about $79 \mathrm{kHz}$. The drop in luminosity over the 10 hour store is due to intra-beam scattering which causes emittance growth. A first upgrade of the RHIC luminosity by a factor of four can be achieved by doubling the number of bunches to about 120 and by increasing focussing at the interaction regions to reduce the beta function by $50 \%$.

Further upgrade of the luminosity requires that the emittance growth from intrabeam scattering is reduced or eliminated. Presently studies have begun to investigate the possibility to use high energy electron beams to cool the gold beams in RHIC. The electron beam intensity required for such a cooler are quite high and will require a high degree of recovery of the beam power. This could be accomplished using a superconducting accelerating/decelerating structures.

It may also be useful to explore the possibility of colliding uranium beams in RHIC. The production of uranium beams may require a completely new preinjector set-up consisting of an Electron Beam Ion Source (EBIS) to produce the highly charged uranium beam and a RFQ and LINAC to accelerate the ions to the Booster injection energy. Development and design of such a new and more versatile source is under way at BNL[1,2].

\section{REFERENCES}

1. Alessi, J., "The RHIC Injector Proposal" in these proceedings.

2. Beebe, E., "Design Issues for the RHIC EBIS" in these proceedings. 